\title{
Elaboración de dosímetros de alanina y sus posibles aplicaciones en riesgos profesionales
}

\author{
Isabel-Cristina Castro ${ }^{1}$, Esperanza Castellanos ${ }^{2}$, Elizabeth Gil ${ }^{3}$, Ovidio Almanza ${ }^{4}$, Gustavo Barreto ${ }^{2}$, \\ José Milciades-Díaz ${ }^{5 *}$
}

\begin{abstract}
${ }^{1}$ Departamento de Microbiología. ${ }^{2}$ Centro Javeriano de Oncología. ${ }^{3}$ Departamento de Química, Facultad de Ciencias, Pontificia Universidad Javeriana. ${ }^{4}$ Departamento de Física. Universidad Nacional de Colombia. ${ }^{5}$ Departamento de Física, Facultad de Ciencias, Pontificia Universidad Javeriana. Bogotá, D.C., Colombia

*jmdiaz@javeriana.edu.co
\end{abstract}

Recibido: 14-01-08; Aceptado 14-08-2009

\begin{abstract}
Resumen
Objetivo. Desarrollar dosímetros de alanina y alanina/parafina con miras a atender la creciente utilización de la radiación ionizante a nivel tecnológico. Materiales y métodos. Los radicales libres producidos por la radiación ionizante en la alanina se detectan por resonancia magnética electrónica (ESR); la parafina se utiliza como aglutinante. La metodología de preparación incluye: elaboración, irradiación, lectura y análisis de datos para los dos tipos de dosímetros. Los espectros ESR estudiados corresponden a dosímetros de alanina con masas entre 60 y $120 \mathrm{mg}$ e irradiados con dosis de 10 y $20 \mathrm{~Gy}$, y dosímetros de alanina/parafina de $160 \mathrm{mg}$ e irradiados con diferentes dosis en un haz de fotones de 4 MV. Resultados. La intensidad de los espectros (las cinco líneas características) depende de las dosis recibidas por los dosímetros y su relación es lineal. Con alanina pura y una dosis de 10 Gy la mínima cantidad requerida fue 120 mg; la compactación del cilindro lograda no fue suficiente para evitar la fragmentación parcial del dosímetro. La parafina no tiene señal paramagnética; en los dosímetros de alanina/parafina (cilindros de longitud $13 \mathrm{~mm}$ y diámetro $3.5 \mathrm{~mm}$, en la relación 80:20, y buena dureza) se estudió la respuesta señal ESR-dosis en un rango entre 20 y $120 \mathrm{~Gy}$. Conclusiones. Los cilindros de alanina/parafina fabricados tienen una dureza apropiada para ser manipulados como dosímetros de radiación ionizante; su reproducibilidad y la efectividad en la acumulación de dosis es buena.
\end{abstract}

Palabras clave: dosimetría, radiación ionizante, espectrometría de resonancia magnética electrónica (ESR), dosímetros de alanina, dosímetros de alanina/parafina.

\begin{abstract}
Elaboration of alanine dosimeters and their possible applications in professional risks. Objective. To develop dosimeters of alanine and alanine/paraffin in order to meet the growing use of ionizing radiation at the technological level. Materials and methods. Free radicals produced by ionizing radiation in alanine are detected by electron spin resonance (ESR); paraffin is used as binder. The methodology of preparation includes: elaboration, irradiation, data collection and data analysis for the two types of dosimeters. The ESR spectra studied correspond to alanine dosimeters with masses between 60 and $120 \mathrm{mg}$ and irradiated with doses of 10 and $20 \mathrm{~Gy}$, and alanine/paraffin dosimeters with $160 \mathrm{mg}$ and irradiated with different doses in a beam of photons $4 \mathrm{MV}$. Results. The intensity of the spectra (the characteristic five lines) depends on the doses received by the dosimeters and their relationship is linear. With pure alanine and a dose of 10 Gy, the minimum amount required was $120 \mathrm{mg}$; the achieved cylinder compaction was not sufficient to avoid the partial fragmentation of the dosimeter. Paraffin has no paramagnetic signal; in the alanine/paraffin dosimeters (cylinders of $13 \mathrm{~mm}$ in length and $3.5 \mathrm{~mm}$ of diameter, with a ratio 80:20, and good hardness) the response signal ESR-dose was studied in a range between 20 and 120 Gy. Conclusions. The cylinders of alanine/paraffin manufactured have an appropriate hardness to be handled as dosimeters of ionizing radiation; their reproducibility and effectiveness in the accumulation of dose is good.
\end{abstract}

Key words: dosimetry, ionizing radiation, spectrometry of electron spin resonance (ESR), alanine dosimeters, alanine/paraffin dosimeters. 


\section{Resumo}

Elaboração de dosímetros de alanina e suas possíveis aplicações em riscos profissionais. Objetivo. Desenvolver dosímetros de alanina e alanina/parafina com a finalidade de satisfazer a crescente utilização de radiações ionizantes ao nível tecnológico. Materiais e métodos. Os radicais livres produzidos pela radiação ionizante na alanina são detectados por ressonância magnética eletrônica (EPR); a parafina é utilizada como aglutinante. O método de preparação inclui: elaboração, irradiação, leitura e analise dos dados para os dois tipos de dosímetros. Os espectros ESR estudados correspondem a dosímetros de alanina com massas entre 60 e 120 mg e irradiados com doses de 10 e 20 Gy, e dosímetros de alanina/parafina de 160 mg e irradiados com diferentes doses de um feixe de fótons de 4 MV. Resultados. A intensidade dos espectros (as cinco linhas características) depende das doses recebidas pelos dosímetros e sua relação é linear. Com alanina pura e uma dose de 10 Gy a mínima quantidade requerida foi de $120 \mathrm{mg}$; a compactação do cilindro alcançada não foi suficiente para evitar a fragmentação parcial do dosímetro. A parafina não tem sinal paramagnético; nos dosímetros de alanina/parafina (cilindros de 13 mm de comprimento e 3,5 mm de diâmetro, na proporção 80:20 e boa dureza) foi estudada a resposta a sinal ESR-dose em um intervalo entre 20 e 120 Gy. Conclusões. Os cilindros de alanina/parafina fabricados têm uma dureza adequada para ser tratados como dosímetros de radiação ionizante; sua reprodutibilidade e eficiência no acúmulo de dose é bom.

Palavras chave: dosimetria, radiação ionizante, espectrometria de ressonância magnética eletrônica (EPR), dosímetros de alanina, dosímetros de alanina/parafina.

\section{Introducción}

El uso intensivo de dosis altas de radiación ionizante en la industria (esterilización de productos médicos, tratamiento de polímeros, tratamiento de alimentos), y en el tratamiento de pacientes con cáncer, han inducido y promovido una constante investigación de sistemas dosimétricos adecuados tanto para la medición de la dosis aplicada en productos irradiados, como en órganos críticos en pacientes. Adicionalmente, se estudia la aplicación de dichos sistemas dosimétricos para la evaluación de la dosis recibida por trabajadores en posibles situaciones accidentales. La dosimetría es la medida de dosis impartida por la energía de la radiación ionizante a la materia. Los dosímetros son los medios empleados para la determinación de tal dosis. Existen diversas clases de dosimetría, esta clasificación depende en esencia de la energía involucrada en el proceso de irradiación y de su necesidad de aplicación.

El concepto de radiación implica transporte de energía. Sin embargo, no cualquier tipo de radiación, al interactuar con estructuras atómicas, puede provocar fenómenos de ionización. Las radiaciones ionizantes, de manera similar a la materia, también presentan una estructura discreta consistente en partículas o fotones y sólo pueden inducir ionizaciones si cada una de estas partículas o fotones posee la energía suficiente (1). Teniendo en cuenta que la energía necesaria para causar el escape de un electrón de valencia en un átomo está en el rango de 4 a 25 eV, radiaciones que posean energía cinética que exceda esta magnitud pueden ser llamadas ionizantes.

Los organismos vivos presentan diversos grados de vulnerabilidad a la energía absorbida y existen evidencias de efectos adversos sobre la salud de las personas expuestas a algunas de estas radiaciones (1). Por tal razón, la adecuada interpretación de los resultados provenientes de la dosimetría, se basa en el adecuado conocimiento de los instrumentos de medida, su apropiado manejo, mantenimiento y calibración, lo cual proporciona la fiabilidad de las medidas realizadas.

En el presente trabajo se estudió la utilización del aminoácido L-alanina como dosímetro de radiación. Los radicales libres inducidos por la radiación en este aminoácido se detectan por medio de la espectroscopia de resonancia magnética electrónica (ESR); el espectro ESR obtenido de la muestra de alanina tiene cinco líneas y la señal se toma como la altura pico a pico de la línea central; como la concentración de radicales libres es función de la dosis absorbida la sustancia puede servir como material dosimétrico (2).

\section{Materiales y métodos}

La metodología para la preparación de los dosímetros, la caracterización del sistema dosimétrico y el desarrollo de los procedimientos de medida para la evaluación de la dosis de radiación ionizante incluyó las siguientes etapas: elaboración, irradiación, lectura y análisis de datos para dosímetros de alanina y alanina/parafina.

\section{Fabricación de dosímetros de alanina y alanina/parafina}

Los dosímetros se elaboraron utilizando L-alanina con un grado del 99\% de pureza, producida por Panreac Química 
S.A., y parafina tipo comercial para la elaboración de dosímetros con mezcla de alanina y parafina.

Para establecer la cantidad mínima de alanina requerida en la fabricación del dosímetro, se prepararon muestras de alanina que fueron medidas por ESR antes y después de ser irradiadas con un haz de fotones de $4 \mathrm{MV}$ y una dosis de 10 Gy. La ausencia de señal de la parafina se comprobó mediante la preparación de muestras de solo parafina y su medición por ESR antes y después de ser irradiadas con una dosis de 10 Gy.

Posteriormente la alanina se maceró y se pasó por un tamiz, malla 400. Para optimizar la cantidad necesaria de alanina y de parafina se consideraron estudios previos (3, 4) y los mejores resultados con nuestras condiciones experimentales los obtuvimos con los dosímetros que contenían un $20 \%$ de parafina.

Para hacer barras cilíndricas prensadas (dosímetros) de 3.5 $\mathrm{mm}$ de diámetro y longitudes del orden de $13 \mathrm{~mm}$, de solo alanina y mezcla alanina/parafina, se empleó un dispositivo cilíndrico de acero plata con siete orificios, dos de guía y cinco para las barras. Para compactar y dar forma a los dosímetros se utilizó una prensa hidráulica. Para retirar los dosímetros prensados del pastillador se usó un dispositivo adicional.

Se elaboraron aproximadamente 100 dosímetros de alanina/parafina y 30 de alanina pura, de los cuales se escogieron aleatoriamente varios grupos para el estudio.

\section{Irradiación de dosímetros de alanina y alanina/parafina}

La irradiación de los dosímetros de alanina y alanina/ parafina, fue realizada en el Centro Javeriano de Oncología, con un acelerador lineal ELEKTA con un haz de fotones de $4 \mathrm{MV}$. Se irradiaron dosímetros de alanina pura con masas entre 60 y $120 \mathrm{mg}$ con dosis de 10 y 20 Gy. A su vez, se irradiaron dosímetros de alanina/parafina con masas del orden de $160 \mathrm{mg}$, los cuales fueron sometidos a dosis de radiación gamma en un rango entre 20 y 120 Gy por sesión.

\section{Lectura de dosímetros de alanina y alanina/ parafina por ESR}

La lectura de los dosímetros se llevó a cabo por espectroscopia de resonancia magnética electrónica (ESR). Se evaluó la respuesta para la caracterización de la señal
ESR, en el espectrómetro Bruker Electron Spin Resonance ESP 300 del Laboratorio de Resonancia del Departamento de Física de la Universidad Nacional de Colombia, sede Bogotá.

Los espectros ESR fueron adquiridos con una fuente de microondas ER041MR, un controlador de microondas $E R 048 H$ y un controlador de campo ER 032M. La lectura de los dosímetros de alanina y alanina/parafina se efectuó en Banda X. Los parámetros utilizados fueron los siguientes: campo central $3358 \mathrm{G}$, ancho de barrido $140 \mathrm{G}$, frecuencia de las microondas $9.42 \mathrm{GHz}$, potencia de las microondas $20 \mathrm{~mW}$, amplitud de modulación $97.8 \mathrm{G}$, frecuencia de modulación $100 \mathrm{kHz}$, ganancia 3.20 × 105 , tiempo total de barrido $41.94 \mathrm{~s}$.

\section{Resultados y discusión}

En relación con la masa óptima del dosímetro, las muestras con masas de 60, 80 y $100 \mathrm{mg}$ mostraron una señal con baja relación señal-ruido $(\mathrm{S} / \mathrm{N})$, de tal forma que no es posible reconocer los cinco picos característicos que presenta el espectro ESR de alanina para una dosis de $10 \mathrm{~Gy}$. A partir de $120 \mathrm{mg}$ el espectro ESR muestra los cinco picos característicos, observándose sólo cambios en intensidad de la señal y no en la resolución del espectro; sin embargo, la presencia de ruido sigue siendo evidente, lo cual se debe a que la dosis se encuentra en el límite de detección del espectrómetro BRUKER ESP 300, por tanto, $120 \mathrm{mg}$ corresponden a la masa óptima para la elaboración del dosímetro (Figura 1).

\section{Dosímetros de alanina}

Por otra parte, teniendo en cuenta que un dosímetro tiene que ser de fácil manejo, los dosímetros de alanina no presentaron las características esperadas debido a que la manipulación de la alanina resultó difícil. Los dosímetros obtenidos resultaron muy frágiles, puesto que se destruían fácilmente al transportarlos al sitio de irradiación y al lugar de medida, manipularlos con pinzas e introducirlos en los tubos de ESR.

\section{Dosímetros de alanina/parafina}

La norma ASTM (6), recomienda el uso de un aglutinante para optimizar la resistencia del dosímetro. El aglutinante elegido fue parafina tipo comercial. Para comprobar la ausencia de señal de la parafina, el espectro ESR correspondiente a la muestra de parafina sin irradiar y a la muestra de 


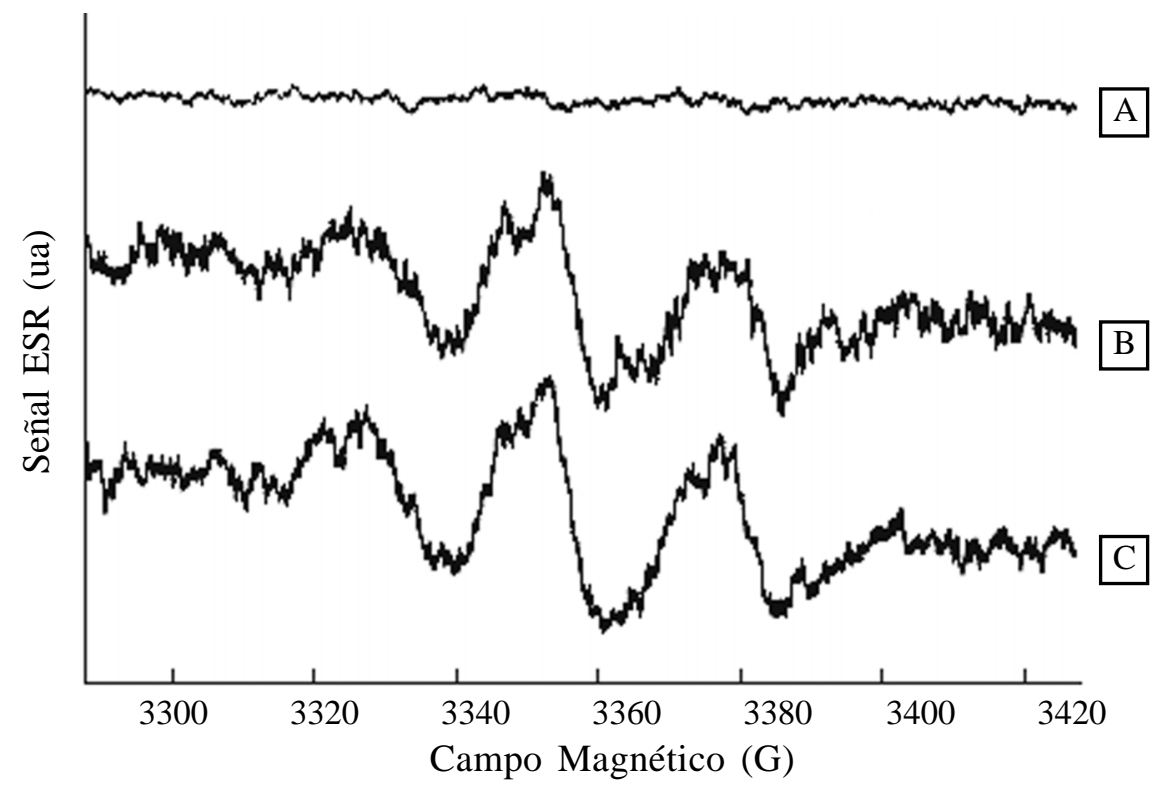

Figura 1. Espectros ESR de muestras de alanina en polvo irradiadas con $10 \mathrm{~Gy}$. A) $60 \mathrm{mg}$, B) $120 \mathrm{mg}$, C) $200 \mathrm{mg}$.

parafina irradiada con una dosis de 10 Gy, no mostró señal. Al comprobar la ausencia de señal de la parafina, se puede afirmar que es apta para ser usada como aglutinante en la fabricación de dosímetros de alanina, debido a que no presenta interferencias respecto a la señal de la alanina.

Se realizaron ensayos previos para determinar las relaciones alanina/parafina adecuadas para el dosíme- tro, obteniéndose los mejores resultados para los dosímetros que contenían una proporción 80:20 alanina/parafina. Los dosímetros obtenidos mejoraron las características mecánicas respecto a los dosímetros de alanina pura, por lo cual mejoró la forma del dosímetro y resulto más fácil su manipulación. El espectro típico de los dosímetros alanina/ parafina se ilustra en la Figura 2.

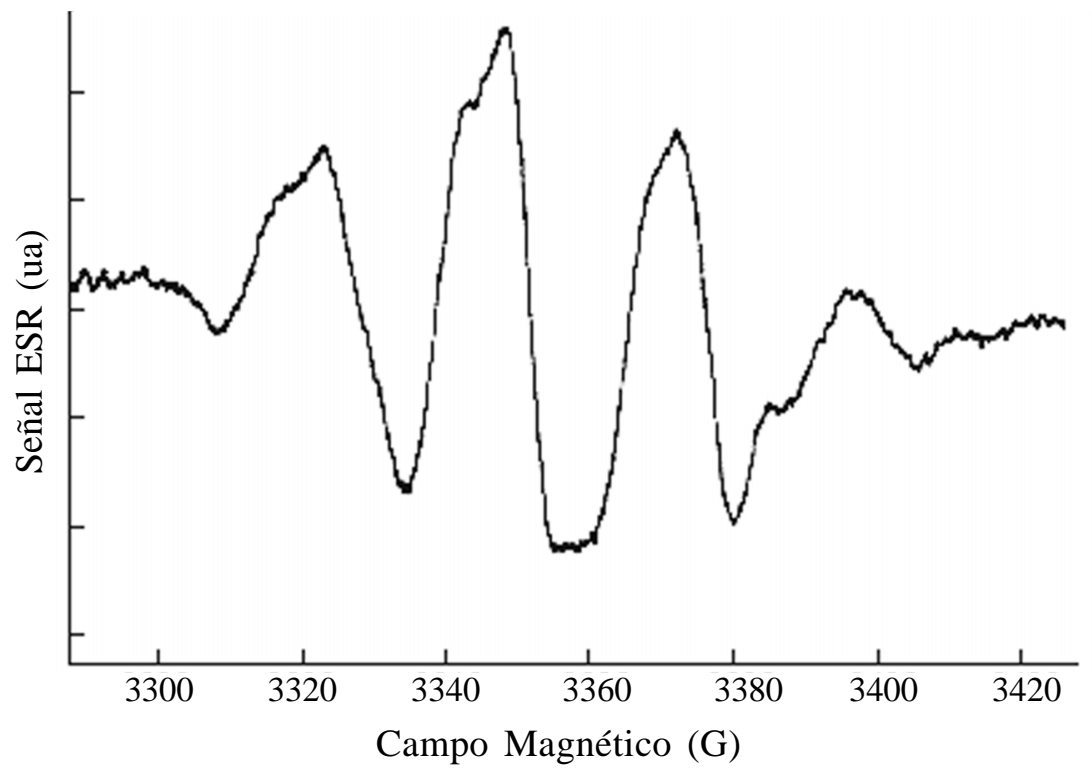

Figura 2. Espectro ESR correspondiente a un dosímetro de alanina/parafina irradiado con 120 Gy. 
La relación señal-ruido ( $\mathrm{S} / \mathrm{N})$ obtenida para los dosímetros irradiados con una dosis de 120 Gy aumentó significativamente, lo que quiere decir que cuando la dosis es mayor la concentración de radicales libres generados por la radiación ionizante también aumenta, incrementando así la resolución y por ende la definición de los picos como se muestra en la figura 2.

Se realizó un estudio dosimétrico de reproducibilidad y de respuesta señal-dosis para los dosímetros de alanina/ parafina. Cuatro grupos de cuatro dosímetros cada uno se irradiaron con dosis (en Gy) así: (Grupo 1: 20, 40, 80 y 120); (Grupo 2: 40, 80 y 120); (Grupo 3: 80 y 120) y (Grupo 4: 120) para obtener 4 dosímetros irradiados con 20 Gy, 8 dosímetros irradiados con 40 Gy, 12 dosímetros irradiados con 80 Gy y 16 dosímetros irradiados con 120 Gy.

En cuanto a la reproducibilidad se obtuvo que a medida que aumentó la dosis de radiación, proporcionalmente aumentó la desviación estándar y el coeficiente de variación de los dosímetros estudiados, comportamiento que debería ser contrario, puesto que a mayor dosis de radiación, se forma mayor número de radicales y por ende aumenta la altura pico a pico.

Teniendo en cuenta que las muestras presentan una respuesta lineal a la dosis de radiación, la respuesta señaldosis para los dosímetros de alanina/parafina, en la proporción 80:20, se estableció mediante una recta de re- gresión lineal, de la altura pico a pico en función de la dosis de radiación (Figura 3 ).

La recta de regresión lineal muestra un coeficiente de variación de 0,99 lo que quiere decir que el modelo de regresión lineal se ajusta muy bien en el rango de dosis estudiado. Como sólo cuatro dosímetros fueron irradiados con una sola dosis, se comprobó la efectividad del dosímetro de alanina/parafina en la acumulación de dosis.

\section{Conclusiones}

Se fabricaron cilindros de alanina/parafina en la proporción 80:20, de $13 \mathrm{~mm}$ de longitud, $3.5 \mathrm{~mm}$ de diámetro con propiedades mecánicas apropiadas para la manipulación como dosímetros de radiación ionizante y se estudió la respuesta señal ESR-dosis en el rango de 20 a 120 Gy, su reproducibilidad y la efectividad de estos dosímetros en la acumulación de dosis.

\section{Financiación}

Investigación financiada por la Fundación para la Promoción de la Investigación y la Tecnología. (Proyecto 2.036).

\section{Conflictos de intereses}

Los autores expresan que no existen conflictos de intereses

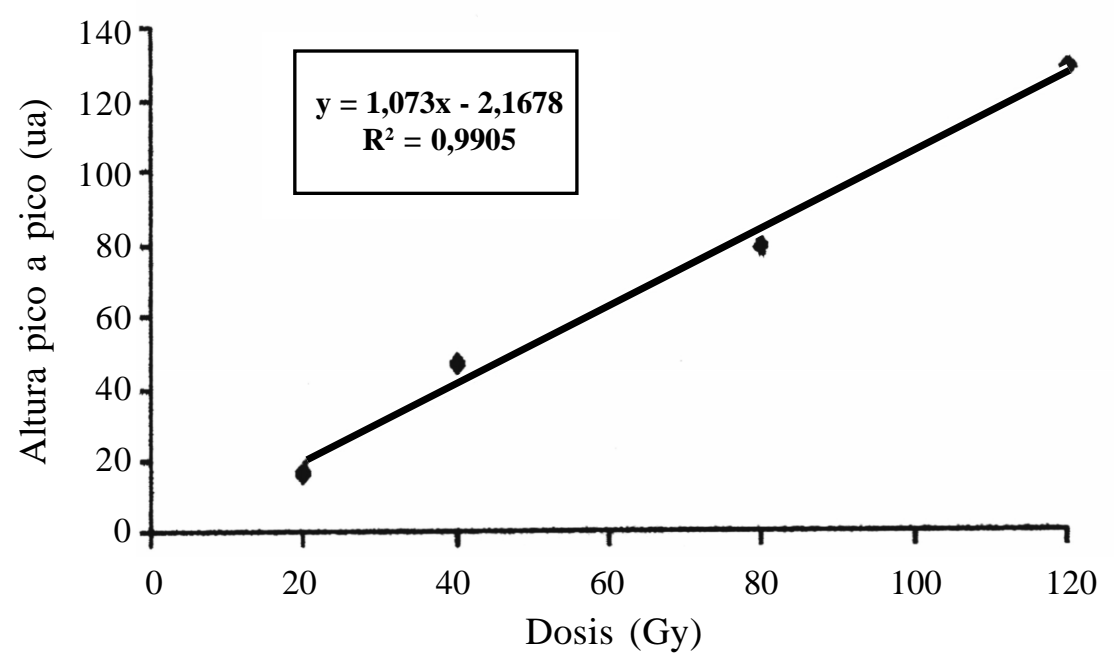

Figura 3. Respuesta señal-dosis para los dosímetros de alanina/parafina. 


\section{Referencias}

1. Falagan Rojo MJ, Alonso Canga A, Ferrer Piñol P, Fernández Quintana JM.. Manual básico de prevención de riesgos laborales: higiene industrial, seguridad y ergonomía. Sociedad Asturiana de Medicina y Seguridad en el Trabajo y Fundación Médicos Asturias. Primera edición: 2000; 165-191.

2. Regulla DF, Deffner U. Dosimetry by ESR spectroscopy of alanine. Applied Radiation. Isotopes. 1982; 33: $1101-1114$.

3. Sleptchonok O, Nagy V, Desrosiers M. Advancements in accuracy of the Alanine dosimetry system. Part 1.
The effects of environmental humidity. Radiation Physics and Chemistry 2000; 57: 115-133.

4. Bergstrand Evas S, Bjerkeb H, Hole Eli O. An experimental investigation of the electron energy dependence of the EPR alanine dosimetry system. Radiation Measurements 2005; 39, 21-28.

5. American Society for Testing and Materials. 2007. Standard Practice for Use of the Alanine-EPR Dosimetry System. Designation E 1607 - 96e1: 1-7. 